\title{
Segmentation for Iris Localisation: A Novel Approach Suitable for Fake Iris Detection
}

\author{
Bodade Rajesh M. ${ }^{1}$ and Talbar Sanjay N. ${ }^{2}$ \\ ${ }^{1}$ Military College of Telecommunication Engineering, Mhow-453441, India \\ rajeshbodade@gmail.com \\ ${ }^{2}$ S.G.G.S. Institute of Engineering and Technology, Vishnupuri, Nanded, India \\ sntalbar@yahoo.com
}

\begin{abstract}
In iris recognition system, accurate iris segmentation and localisation from eye image is the foremost important step. In this paper a robust and efficient method of iris segmentation is proposed. In the proposed method, the outer boundary of iris is calculated by tracing objects of various shape and structure. Based on the pupil size variation, the inner boundary of iris is detected. The variation in pupil size is also used for aliveness detection of iris. Thus, this approach is a very promising technique in making iris recognition systems more robust against fake-iris-based spoofing attempts. The algorithm is tested on UPOL database of 384 images both eyes of 64 subjects. The success rate of accurate iris localisation from eye image is $99.48 \%$ with minimal loss of iris texture features in spatial domain as compared to all existing techniques. The processing time required is also comparable with existing techniques.
\end{abstract}

Keywords: Iris Segmentation, Fake Iris Detection, Pupil Dynamics, Dynamic Iris Localisation.

\section{Introduction}

Identification of people is getting more and more importance in the increasing network society. Biometrics is the branch of science in which human beings are identified with their behavioral or physical characteristics. Among all biometrics, iris recognition is the gaining more attention because iris of every person is unique and it never changes during a person's lifetime [1-8].

The acquired image of eye does not contain only iris but it also contains pupil and data derived from the surrounding eye region like sclera, eyelid and eyelashes. Therefore, it is extremely important to segment and localize the iris from the acquired eye image, prior to feature extraction. Thus, the overall performance of iris recognition system is decided by the fact that how accurate iris is segmented and localized from an eye image[1].

This paper explains the novel approach of dynamic iris localisation based on comparison of two images of different intensities for complete and accurate localisation of iris without any loss of iris features. 
The reminder of this paper presents Related work, Proposed system, Outer boundary detection, Inner boundary detection, Experimental results and Conclusions in Sections 2, 3, 4, 5, 6 and 7 respectively.

\section{Related Work}

A generalised iris recognition consists of image acquisition, iris segmentation and localization (preprocessing), feature extraction and feature comparison (matching). Biometric based personal identification using iris requires accurate iris localization from an eye image [2]. Several researchers have implemented various methods for segmentation and localising the iris. John Daugman [3] has proposed one of the most practical and robust methodologies, constituting the basis of many functioning systems. He used integro-differential operator to find both the iris inner and outer boundaries for iris segmentation. Wildes [2] proposed a gradient-based binary edge map construction followed by circular Hough transform for iris segmentation. Several researchers have proposed several variants of these methods with minor variations in the research paperes [5-8].

Almost all methods stated are based on the assumption that centre of iris (Outer Boundary) and Pupil (Inner boundary) is same and iris is perfectly circular in shape which are seldom true. Therefore, the iris segmentation and localization from an acquired image leads to loss of texture data near to pupil and/or outer boundary.

\section{Proposed System of Iris Localisation}

Iris possess high degree of randomness with high variation in eye colour contrast and texture, to obtain maximum information and efficiency for recognition, high contrast images are required. Fig.1 shows sample images with low, medium and high contrast from CASIA, UBIRIS and UPOL iris databases respectively[9-11].

Fig. 2 shows the flow chart of proposed method of iris localisation using two iris images at different light levels (Intensities). It mainly consist of three steps, viz., Outer (Iris) Boundary Detection, Inner (Pupil) Boundary Detection and Normalisation.

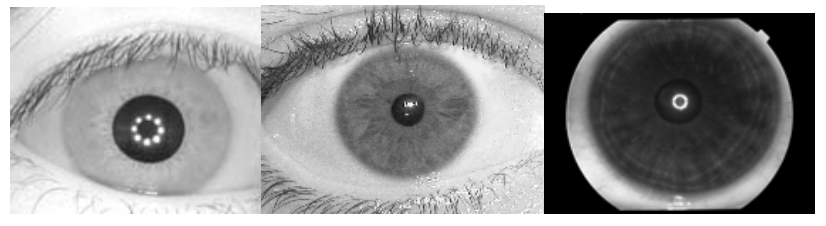

Fig. 1. Low, Medium and High contrast eye images 


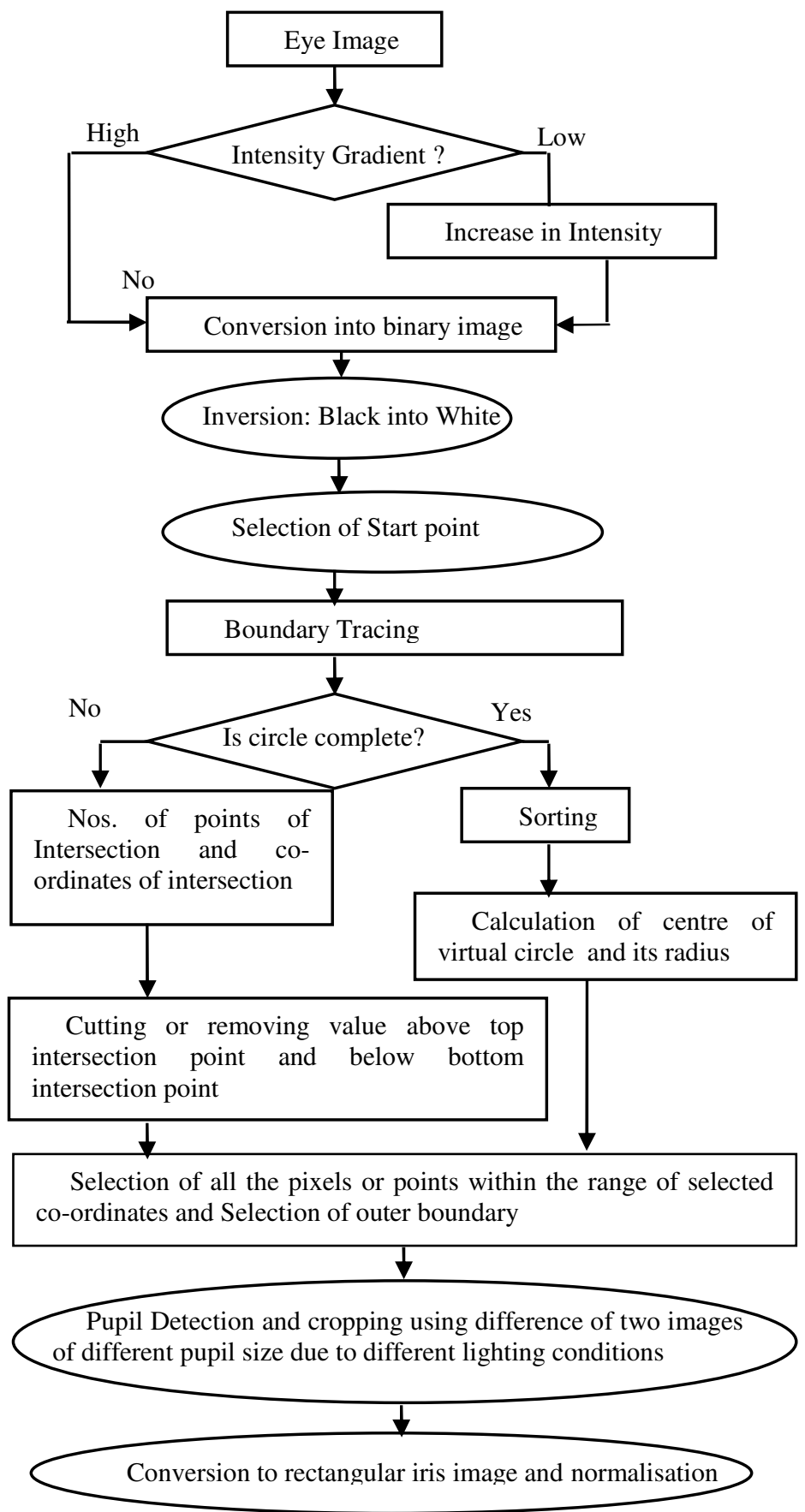

Fig. 2. Flow chart of a proposed system 


\section{Outer Boundary Detection}

Since the high contrast images provides prominent texture information of iris as well as other components of eye, these other components creates problem in iris localization while calculating the threshold value, which is used for converting image into binary images. So to overcome this problem it is required to trace irregular objects with of different shapes and structure. Empirical study of various shows that iris is located generally on central area of image. Tracing can be started from any one corner of image.

Boundary is being traced of all points with binary value as ' 1 ' in all direction starting from selected point that is the first point that has value as ' 0 ' coming from top to bottom in any one quarter of image. Thus complete boundary is traced for a complete iris without any intersection. For images with intersection with upper or lower eyelids, as shown in Fig 3(b) may not result into a complete one object, for such cases point of intersection is calculated and all points above point of intersection in case of intersection with upper eyelid and points below in case of intersection with lower eyelids are removed. The traced boundary of iris is shown in Fig 3(a) with green colour and a virtual circle is drawn using all these traced points with blue colour. In case of complete iris or complete circle is being traced then area under the green colour boundary is trace else in case of intersection objects within virtual circle with blue colour boundary is selected. This selected area is segmented from rest of the image.

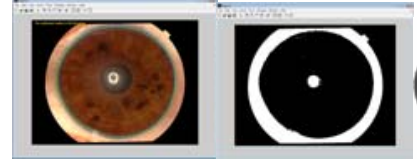

(a)
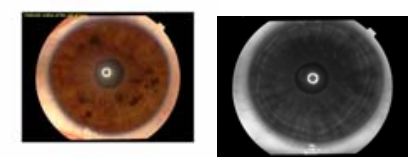

(b)

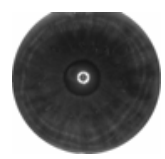

Fig. 3. Outer Boundary detection in case of (a) complete circle and (b) incomplete circle

\section{Inner Boundary (Pupil) Detection}

Selection of inner boundary of iris is one of the most complicated part of iris localisation. Thus in this report a novel approach is used, in which, two images of iris are taken at different light intensities and the variation in intensity of light will produce variation in size of pupil[3-4] thus taking images of iris at these two light intensities will give similar images but with variation in size of pupil.

Now comparing or subtracting these two images. As iris part of two images is same, result of subtraction will give 0 value and only place where non zero values are obtained is the region of pupil due to variation in size of pupil. The resultant image will also have number of visible spots as noise which are required to be remove to calculate exact size of pupil.

Size of pupil is larger than the size of obtained noise, converting this image into binary image gives complete iris of smaller pupil along with noise, on removing all the objects of smaller size than the predefined value results into exact inner boundary of pupil as shown in Fig 4. 


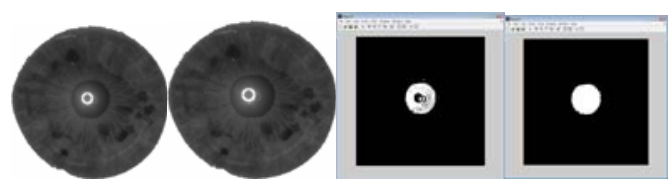

Fig. 4. Detection of Inner (Pupil) Boundary

Tracing this inner boundary and selecting region outside inner boundary and below outer boundary will give exact iris with minimum losses as shown in Fig 5(a). Finally, completely detected iris is converted to rectangular image using normalization Equation (1) and (2) as shown in Fig 5(b).

$$
\begin{aligned}
& \mathrm{x}_{1}=\mathrm{x}+\mathrm{r} * \cos (\Phi) \\
& \mathrm{y}_{1}=\mathrm{y}+\mathrm{r} * \sin (\Phi)
\end{aligned}
$$

where, $(\mathrm{x}, \mathrm{y})$ are the coordinates of center of the ring, $\left(\mathrm{x}_{1}, \mathrm{y}_{1}\right)$ are the coordinates of pixel of rectangular image, $r$ is a radius of iris ring that varies form inner to outer boundary of iris image and $\Phi$ is an angle of that varies from 0 to 360 degree.

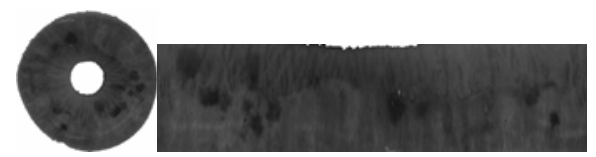

Fig. 5. (a) Segmented Iris (b) Normalised rectangular Iris

\section{Results}

The database contains $3 \times 128$ iris images (i.e. $3 \times 64$ left and $3 \times 64$ right). The images are: 24 bit - RGB, 576 x 768 pixels, file format: PNG. The irises were scanned by TOPCON TRC50IA optical device connected with SONY DXC-950P 3CCD camera[17]. The proposed algorithm is implemented in MATLAB7.0, on PIV-3Ghz, Intel processor with 512MB RAM and tested on UPOL database[11].

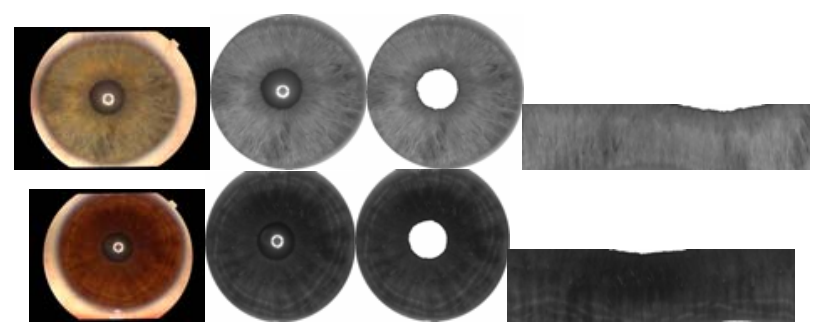

Fig. 6. Iris localisation output for a few eye images of the database

Fig 6 shows the output of various stages of algorithm for sample images of database. The segmentation accuracy and timing analysis of the algorithm and its comparison with existing algorithms is given in Table 1. 
Table 1. Result of segmentation accuracy and timing analysis

\begin{tabular}{|c|c|c|}
\hline Methodology & Accuracy & Time in Seconds \\
\hline Proposed & $99.48 \%$ & 1.43 \\
\hline Daugman[3] & $67.23 \%$ & 1.03 \\
\hline Wildes[2] & $88.49 \%$ & 1.3 \\
\hline Masek[7] & $83.97 \%$ & 7.8 \\
\hline Narote[8] & $91.33 \%$ & 1.21 \\
\hline
\end{tabular}

The experimental results have shown that the proposed algorithm gives better results. The algorithm accurately extracted irises of 382 images out of 384 images of 64 subjects giving a success rate of $99.48 \%$ that very minimal loss of iris texture features as compared to existing methods, specially for high contract iris images.

\section{Conclusions}

The proposed method showed the very high accuracy rate of iris segmentation and at comparable timing cost.

The strength of the method is that it does ont assume the centre of pupil and centre of iris as same as against other method and hence it is more practical one.

Moreover, the method is based on the comparison of two iris images at different light levels to detect the change in the sixe of pupil. Thus, this is a very promising technique for making iris recognition systems more robust against fake-iris-based spoofing attempts as well. This makes this method more useful than any other methods.

We are extending the use of this method for fake iris detection / aliveness detection of iris for full-proof iris recognition system with inherent anti-spoofing mechanism[4].

\section{References}

1. Kong, W., Zhang, D.: Accurate iris segmentation based on novel reflection and eyelash detection model. In: Proceedings of 2001 International Symposium on Intelligent Multimedia, Video and Speech Processing, Hong Kong, May 2001, pp. 263-266 (2001)

2. Wildes, R.: Iris Recognition: An Emerging Biometric Technology. Proc. IEEE 85, 13481363 (1997)

3. Daugman, J.: How iris recognition works. IEEE Transactions on Circuits and Systems for Video Technology 14, 21-30 (2004)

4. Daugman, J.: Anti-spoofing Liveness Detection, http://www.cl.cam.ac.uk/users/igdl000/countermeasures.pdf

5. Boles, W., Boashash, B.: A Human Identification Technique Using Images of the Iris and Wavelet Transform. IEEE Trans. Signal Processing 46, 1185-1188 (1998)

6. Ma, L., Wang, Y., Tan, T.: Personal Identification Based on Iris Texture Analysis. IEEE Trans. on PAMI 25(12), 414-417 (2003) 
7. Masek, L., Kovesi, P.: MATLAB source code for a Biometric Identification System Based on Iris Paterns. The school of Computer Science and Software Engineering, The University of Western Austrilia (2003)

8. Narote, S.P., Narote, A.S., Waghmare, L.M.: An automated Segmentation Method for Iris Recognition. In: Proceedings of TENCON 2006. 2006 IEEE Region 10th Conf., November 14-17 (2006)

9. Chinese Academy of Sciences Institute of Automation, Database of 756 Greyscale Eye Images, http: / / www. sinobiometrics.com

10. Proença, H., Alexandre, L.A.: UBIRIS: iris image database (2004), http://iris.di.ubi.pt

11. High contrast Iris image database downloaded from, http://phoenix.inf.upol.cz/iris/download/ 